DIW BERLIN

Discussion

Papers

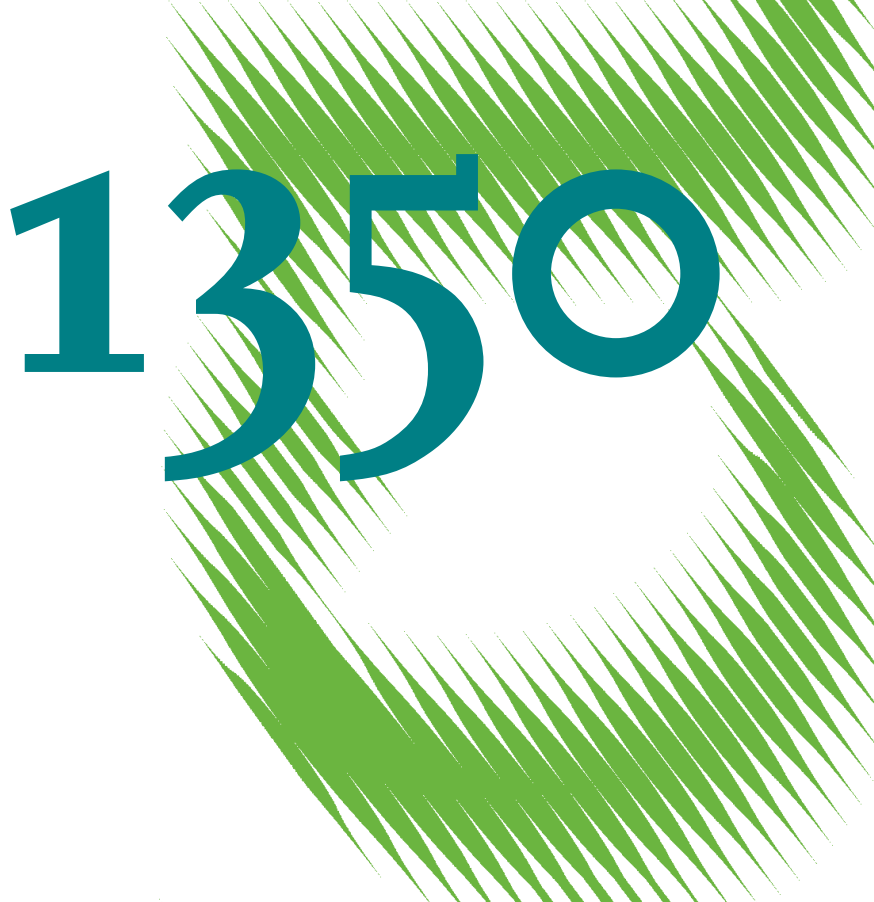

Can Stock Price Fundamentals

Properly be Captured?

Using Markov Switching in Heteroskedasticity Models to Test Identification Schemes 
Opinions expressed in this paper are those of the author(s) and do not necessarily reflect views of the institute.

IMPRESSUM

(C) DIW Berlin, 2013

DIW Berlin

German Institute for Economic Research

Mohrenstr. 58

10117 Berlin

Tel. $+49(30) 89789-0$

Fax +49 (30) $89789-200$

http://www.diw.de

ISSN print edition $1433-0210$

ISSN electronic edition 1619-4535

Papers can be downloaded free of charge from the DIW Berlin website:

http://www.diw.de/discussionpapers

Discussion Papers of DIW Berlin are indexed in RePEc and SSRN:

http://ideas.repec.org/s/diw/diwwpp.html

http://www.ssrn.com/link/DIW-Berlin-German-Inst-Econ-Res.html 


\title{
Can Stock Price Fundamentals Properly be Captured? Using Markov Switching in Heteroskedasticity Models to Test Identification Schemes
}

\author{
November 2013 \\ Anton Velinov \\ DIW Berlin and European University Institute, Florence \\ Mohrenstr. 58, D-10117 Berlin, Germany \\ email: avelinov@diw.de \\ tel. +4917627774345
}

\begin{abstract}
Structural identification schemes are of essential importance to vector autoregressive (VAR) analysis. This paper tests a commonly used structural parameter identification scheme to assess whether it can properly capture fundamental and non-fundamental shocks to stock prices. In particular, five related structural models, which are widely used in the literature on assessing stock price determinants are considered. They are either specified in vector error correction (VEC) or in VAR form. Restrictions on the long-run effects matrix are used to identify the structural parameters. These identifying restrictions are tested by means of a Markov switching in heteroskedasticity model. It is found that for two of the five models considered, the long-run identification scheme appropriately classifies shocks as being either fundamental or non-fundamental. A series of robustness tests are performed, which largely confirm the initial findings.
\end{abstract}

Key Words: Markov switching model, vector autoregression, vector error correction, heteroskedasticity, stock prices

JEL classification: C32 C34 


\section{Introduction}

The question of how well stock prices reflect their underlying economic fundamentals has been widely studied in the literature. A popular approach to answer this question is to make use of vector autoregressive (VAR) or vector error correction (VEC) models. An essential step of such models is to set up appropriate restrictions so as to identify the structural shocks of interest. Only based on correctly identified structural shocks, one is able to answer the question of whether stock prices are mainly driven by their fundamentals or by non-fundamental factors.

However, issues regarding identifying restrictions are seldom addressed in the literature on stock price determinants. This paper is the first to do that in this line of literature. In fact there are several popular structural models, using a similar type of identification scheme, which claim to identify fundamental and non-fundamental (bubble) components of stock prices. The models along with the papers making use of them are summarized in Table 1 . All these models are similar in the sense that they can be derived from asset pricing theory; the price of an asset being equal to its future discounted cash flows (for example dividends) 1

As is common in the literature, all of the models in Table 1 make use of long-run restrictions as in Blanchard and Quah (1989) to identify the structural shocks. In fact, it is always the case that a lower triangular identification scheme is employed. This paper aims to test whether this identification scheme is supported by the data so as to be able to shed some light on the issue of whether stock price determinants have properly been captured. In other words, this analysis is only focused on determining whether the structural parameter identification scheme is supported by the data.

We test the structural identifying restrictions by using the method developed in Lanne et al. (2010) and in Herwartz and Lütkepohl (2011). Both extend the basic multivariate structural model to allow for switching covariance matrices according to a Markov process. This allows for heteroskedastic error terms across states. Due to the extra covariance parameters, any structural restrictions then become over-identifying and can thus be tested. This procedure is explained in detail in section 2 along with the relevant models used.

This paper finds support for the structural identification scheme for two out of the five models listed in Table 1 . Those are the models with real GDP and real dividends as proxies of real economic activity. Results of this paper show that identifying restrictions need to be implemented with an eye on the type of data one is using as a proxy for the relevant economic

\footnotetext{
${ }^{1}$ The dividend discount model (DDM) is popular in asset pricing. Its basic premise is that an asset's price is the sum of its expected future discounted payoffs (i.e. dividends). These payoffs are necessarily linked to real economic activity such as real GDP or industrial production. Hence, Table 1 can be thought of as summarizing multiple variants of the popular asset pricing model.
} 
Table 1: Popular models used in the literature.

\begin{tabular}{|c|c|}
\hline (Subset) Model & Used by \\
\hline \multirow[t]{2}{*}{$y_{t}=\left[Y_{t}, r_{t}, s_{t}\right]^{\prime}$} & Lee (1995a), Rapach $(2001)^{*}, \overline{B i n s w a n g e r}$ 2004b), Jean and Eldomiaty $(2010)$ \\
\hline & Lanne and Lütkepohl $\mid$ (2010) \\
\hline$y_{t}=\left[I P_{t}, r_{t}, s_{t}\right]^{\prime}$ & Binswanger 2004b), Laopodis 2009, \\
\hline$y_{t}=\left[D_{t}, r_{t}, s_{t}\right]^{\prime}$ & Lee (1995a), Allen and Yang (2004), Jean and Eldomiaty [2010) \\
\hline$y_{t}=\left[E_{t}, r_{t}, s_{t}\right]^{\prime}$ & Binswanger (2004b), Jean and Eldomiaty 2010 \\
\hline$y_{t}=\left[E_{t}, D_{t}, s_{t}\right]^{\prime}$ & Lee (1998), Chung and Lee (1998), Binswanger 2004b), Jean and Eldomiaty (2010) \\
\hline
\end{tabular}

Here $Y_{t}, I P_{t}, D_{t}, E_{t}, r_{t}$ and $s_{t}$ stand for real GDP, industrial production index, real dividends, real earnings, real interest rates and real stock prices respectively.

* These variables are a subset of the variables used in the original model.

indicator. For instance, the researcher needs to be sure that the given data series she uses captures the economic concept to be investigated well.

After elaborating on the models, section 3 discusses the estimation and testing procedure briefly. Section 4 presents the model results along with relevant details on the model selection procedure. Section 5 deals with model robustness issues and finally section 6 summarizes the main conclusions.

\section{The Models}

This section briefly sets out the structural vector autoregressive (SVAR) and error correction (SVEC) models that are considered. Later a regime switching extension is introduced and the basic methodology for testing the identifying restrictions is explained.

\subsection{The SVAR and SVEC models}

The basic vector autoregressive model with $p$ lags, $\operatorname{VAR}(p)$ can be written as

$$
y_{t}=v+A_{1} y_{t-1}+A_{2} y_{t-2}+\ldots+A_{p} y_{t-p}+u_{t},
$$

where $y_{t}$ is a $(K \times 1)$ vector of stationary endogenous variables, $v$ is a $(K \times 1)$ vector of constants and $A_{i}, i=1, \ldots, p$ are $(K \times K)$ autoregressive parameter matrices. The $(K \times 1)$ vector of reduced form error terms, $u_{t}$ is assumed to have an expected value of 0 and a positive definite covariance matrix $\Sigma_{u}$. Hence, $u_{t} \sim\left(0, \Sigma_{u}\right)$. 
A reduced form vector error correction model, $(\operatorname{VEC}(p-1))$ is given as follows

$$
\Delta y_{t}=v_{t}+\Pi y_{t-1}+\Gamma_{1} \Delta y_{t-1}+\Gamma_{2} \Delta y_{t-2}+\ldots+\Gamma_{p-1} \Delta y_{t-p+1}+u_{t}
$$

where now $y_{t}$ may include variables with unit roots. Here $v_{t}$ is a $K$ dimensional deterministic component that can include an intercept and a trend term, hence $v_{t}=v_{0}+v_{1} t$. Further, $\Gamma_{i}, i=1, \ldots, p-1$ are $(K \times K)$ parameter matrices and the residual terms, $u_{t}$ are assumed to have the same properties as before. Here $\Delta$ is the first difference operator (so that $\Delta y_{t}=$ $y_{t}-y_{t-1}=(1-L) y_{t}$, where $L$ is the lag operator). This means that $\Delta y_{t}$ is assumed to be $I(0)$, such that $\Pi y_{t-1}$ also needs to be stationary. The $(K \times K)$ matrix $\Pi$ is of rank $r$, (where $0<r<K$ ) and captures the cointegrating relations of the model. More specifically, since $\Pi$ is singular, it can be decomposed into the product of two $(K \times r)$ matrices of full column rank, $\alpha, \beta$ so that $\Pi=-\alpha \beta^{\prime}$. Here $\beta$ is referred to as the cointegrating matrix and contains the $r$ linearly independent cointegrating relations, so that $\beta^{\prime} y_{t-1}$ is stationary, and $\alpha$ is known as the loading matrix.

In line with the literature, structural shocks are defined as $u_{t}=B \varepsilon_{t}$, where $\varepsilon_{t}$ is a $K$ dimensional vector of structural residuals such that $\varepsilon_{t} \sim\left(0, \Sigma_{\varepsilon}\right)$, where $\Sigma_{\varepsilon}$ is usually assumed to be $I_{K}$, the identity matrix. Here $B$ is a $(K \times K)$ matrix depicting contemporaneous effects. According to these assumptions $\Sigma_{u}=B B^{\prime}$. The structural parameters can be derived from the reduced form parameters. However, since $\Sigma_{u}$ is symmetric, this only leaves $K(K+1) / 2$ reduced form parameters to identify the $K^{2}$ structural parameters of the $B$ matrix. Hence, $K^{2}-K(K+1) / 2=K(K-1) / 2$ restrictions need to be imposed. How this is done for each model is discussed in the following.

\subsubsection{Restrictions on the VAR model}

All the papers considered in Table 1 make use of long run identifying restrictions, as in Blanchard and Quah (1989). How such restrictions are imposed is briefly explained here. Rewriting equation (1) in lag polynomial form gives

$$
A(L) y_{t}=v+u_{t} \text {, }
$$

where $A(L)=I_{K}-A_{1} L-A_{2} L^{2}-\cdots-A_{p} L^{p}$. Provided that $A(L)^{-1}$ exists, the Wold moving average (MA) representation for the stationary $y_{t}$ process is

$$
y_{t}=\mu+\sum_{s=0}^{\infty} \Phi_{s} u_{t-s}=\mu+\Phi(L) u_{t},
$$

where $\mu=\left(I_{K}-A_{1}-A_{2}-\cdots-A_{p}\right)^{-1} v=A(1)^{-1} v, \Phi(L) \equiv A(L)^{-1}$ and $\Phi_{0}=I_{K}$. Having defined the structural shocks as $\varepsilon_{t}=B^{-1} u_{t}$, the structural representation of $(4)$ is

$$
y_{t}=\mu+\sum_{s=0}^{\infty} \Psi_{s} \varepsilon_{t-s}=\mu+\Psi(L) \varepsilon_{t},
$$


here $\Psi_{i} \equiv \Phi_{i} B$, for $i=0,1,2, \ldots$. The accumulated long-run effects of the structural shocks over all time periods are given by the long-run impact matrix, $\Psi \equiv \Phi B$, where $\Phi \equiv \sum_{s=0}^{\infty} \Phi_{s}=$ $A(1)^{-1}$. It is on the $\Psi$ matrix that Blanchard and Quah (1989) suggest imposing identifying restrictions, usually in the form of zeros. That way some shocks have permanent effects, while others only have transitory effects.

As is common practice, most papers mentioned in Table 1 make use of the following lower triangular $\Psi$ matrix

$$
\Psi=\left[\begin{array}{ccc}
\star & 0 & 0 \\
\star & \star & 0 \\
\star & \star & \star
\end{array}\right],
$$

where $\star$ denotes an unrestricted element. Depending on the way the variables are arranged this identification scheme distinguishes between fundamental and non-fundamental shocks. The non-fundamental shock is assumed not to have any permanent effect on any of the variables except the last one (last column of (6)). The other two shocks are assumed to be of a fundamental nature; in that one of them (first column of (6) influences all variables in the long-run, while the other (second column of (6)) only leaves a permanent impact on the last two model variables. 2 The identification scheme in 6 is used for testing restrictions on SVAR models throughout this paper.

\subsubsection{Restrictions on the VEC model}

The long-run effects matrix for a VEC model is not derived in such a straightforward way as that for a VAR model. Fortunately, from Granger's representation theorem, the VEC counterpart of $\Phi$ is given as

$$
\Xi=\beta_{\perp}\left[\alpha_{\perp}^{\prime}\left(I_{K}-\sum_{i=1}^{p-1} \Gamma_{i}\right) \beta_{\perp}\right]^{-1} \alpha_{\perp}^{\prime},
$$

where $\perp$ stands for the orthogonal complement of a given matrix. For instance, the orthogonal complement of an $(m \times n)$ matrix, $A$, is given by the $(m \times(m-n))$ matrix, $A_{\perp}$. The $\Xi$ matrix is computed from the estimates of the reduced form parameters. Hence, the long-run impact matrix is $\Xi B$ and restrictions can be imposed on it in a similar way as on the $\Psi$ matrix above.

A quick note on the restrictions of the SVEC model is in order. Since $\Xi$ is a singular matrix, restrictions need to be placed appropriately. In particular, the rank of $\Xi$ is $K-r$ and according

\footnotetext{
${ }^{2}$ The zero restriction in the second column of $\Psi$ in 6 is left out in Lee (1995a) and Laopodis 2009). The shocks are still labeled as fundamental and non-fundamental, even though the model itself is underidentified. Further, the models used in Jean and Eldomiaty (2010) are initially identified according to the Swanson and Granger (1997) identification scheme, however, in a section on model robustness, they mention that a lower triangular long-run impact matrix as in 6 performs equally well.
} 
to King et al. (1992) there can be at most $r$ transitory shocks, i.e. $r$ columns of $\Xi B$ can be 0 and each column of zeros stands for only $K-r$ restrictions. In addition, there need to be $r(r-1) / 2$ restrictions on the $B$ matrix to identify the non-permanent shocks. The remaining restrictions needed to identify the model (exactly) can be placed on the non-zero elements of $\Xi B$ or directly on $B$. A good summary of placing restrictions on a SVEC model can be found in Lütkepohl (2005).

In a similar lower triangular fashion, long-run restrictions on SVEC models in this paper are placed as follows

$$
\Xi B=\left[\begin{array}{ccc}
\star & 0 & 0 \\
\star & \star & 0 \\
\star & \star & 0
\end{array}\right] .
$$

Here again $\star$ denotes unrestricted elements. It is now assumed that a non-fundamental shock does not have permanent effects on any of the other variables, i.e. the last column of 7) contains only zeros. Note that such an assumption cannot be made for the SVAR model restrictions since $\Psi$ in (6) cannot be a singular matrix. It may be more realistic to assume that shocks labeled as non-fundamental do not have a permanent impact on any of the model variables. Further, without skipping too far ahead, the rank of $\Xi$ turns out to be two for all VEC models and hence, the last column of zeros provides two independent restrictions. The identification scheme in (7) is thus enough to just identify the SVEC model in the traditional sense.

\subsection{The Markov switching SVAR and SVEC models}

In order to test identification schemes, as in (6) or in 7), it is necessary to expand the basic model to allow at least for switching covariance matrices. Further, for estimation convenience it is also assumed that residuals are normally distributed, hence,

$$
u_{t} \sim \operatorname{NID}\left(0, \Sigma_{u}\left(S_{t}\right)\right) .
$$

As is made clear in Lanne et al. (2010), the normality assumption in no way limits the unconditional distribution and it is also not a crucial assumption for the analysis. Here $S_{t}$ is assumed to follow a first-order discrete valued Markov process with transition probabilities given by

$$
p_{i j}=P\left(S_{t}=j \mid S_{t-1}=i\right) .
$$

These can be grouped in an $(M \times M)$ matrix of transition probabilities, $P$ such that the rows add up to 1 and where $M$ are the number of different states.

Note that it is also possible to allow for switches in the intercept term, $v$ in the SVAR case and $v_{0}$ in the SVEC case. In principle, all the parameters could be subject to regime switches, 
however such assumptions would need to be justified in the sense of there being structural breaks in the data or some reasonable economic explanation as to why a certain parameter could be switching. In this analysis it is crucial for the covariance matrices to be switching, it may also be reasonable to assume - given the data used - that the intercept parameter could be subject to regime switches as is discussed later. All other parameters are assumed to be stable.

As already noted, the Markov switching (MS) model is a convenient way of dealing with data subject to structural breaks. In the relevant literature changes in structural relationships are documented in Lee (1998), Chung and Lee (1998), Binswanger 2000, 2004a c) and Laopodis (2009) among others. In this sense, a MS model may also be better suited to answering the question of how well stock prices are reflected by their fundamentals. However, in this paper a MS model is used solely to test the above-mentioned identifying restrictions.

\section{Estimation and Testing Procedure}

This section briefly examines the estimation and testing procedures used in this paper. Starting with the VAR model, its parameters are estimated by means of OLS. Since only long-run restrictions are imposed, estimation of the structural parameters is straightforward. With a simple substitution it follows that $\Phi \Sigma_{u} \Phi^{\prime}=\Psi \Psi^{\prime}$. The left hand side of this equation is known, hence for a fully identified model, $\Psi$ is easy to derive. The contemporaneous matrix is then easily obtained as $B=\Phi^{-1} \Psi$.

The VEC parameters are estimated by the method of reduced rank regression in Johansen (1995). Since the cointegrating matrix, $\beta$, is not unique it can be identified by a simple normalization such that the first $r$ rows contain an $(r \times r)$ identity matrix, as is shown in ?. The structural parameters are estimated by an iterative algorithm proposed by Amisano and Giannini (1997) subject to identifying restrictions placed as in Vlaar (2004).

The parameters of the MS models are estimated using the iterative expectation maximization (EM) algorithm. This algorithm was initially popularized by Hamilton (1994) for univariate processes and later extended to multivariate processes by Krolzig (1997). Since the $\beta$ matrix in the VEC models symbolizes long-run relationships, it is not re-estimated at each maximization step of the EM algorithm. It is trivial to change this though, so that a reduced rank regression is performed in each maximization step. However, this merely leads to an increase in computational time without really affecting the conclusions obtained below.

In order to test the identifying restrictions it is necessary to decompose the covariance matrices in the following way

$$
\Sigma_{u}(1)=B B^{\prime}, \quad \Sigma_{u}(2)=B \Lambda_{2} B^{\prime}, \quad \ldots \quad \Sigma_{u}(M)=B \Lambda_{M} B^{\prime} .
$$


This adds to a nonlinear optimization procedure in the maximization step of the EM algorithm. The underlying assumption is that the contemporaneous effects matrix, $B$ stays the same across states. Here the $\Lambda_{i}, i=2, \ldots, M$ matrices are diagonal with positive elements, $\lambda_{i j}, i=2, \ldots, M, j=1, \ldots, K$ and can be interpreted as relative variance matrices. In order for the $B$ matrix in (9) to be unique up to changes in sign and column ordering, it is necessary for all pairwise diagonal elements in at least one of the $\Lambda_{i}, i=2, \ldots, M$ matrices to be distinct. For example, for a 3 -state model it is required that $\lambda_{i j} \neq \lambda_{i l}, i=2$ and/or $3, j, l=1, \ldots, K, j \neq l$. Hence, even if these elements are equal in one state, they should not be equal in the other state. For a more detailed explanation of the uniqueness of the $B$ matrix the reader is referred to Proposition 1 in the appendix of Lanne et al. (2010). If this distinction requirement is fulfilled, then $B$ is said to be identified through heteroskedasticity.

The assumption of an invariant $B$ matrix may seem rather crucial to this analysis. However, when there are more than two states, this assumption can be tested by means of a likelihood ratio (LR) test. The test statistic has an asymptotic $\chi^{2}$ distribution with (1/2) MK $K+$ $1)-K^{2}-(M-1) K$ degrees of freedom. Clearly, if $M=2$ the degrees of freedom would be 0 , thus giving a nonsensical result. Note, that the above procedures closely follow Lanne et al. 2010) and Herwartz and Lütkepohl (2011).

Standard errors of the parameter estimates are obtained from the inverse of the negative of the Hessian matrix evaluated at the optimum. Distinction of the $\lambda_{i j}, i=2, \ldots, M, j=$ $1, \ldots, K$ parameters is then determined through Wald tests. It is also possible to use LR tests for this purpose, however, such tests may not give very accurate conclusions since they can potentially converge to the same optimum each time. 3

If the distinction of the $\lambda_{i j}, i=2, \ldots, M, j=1, \ldots, K$ parameters is fulfilled, the $B$ matrix is identified up to changes in sign and column ordering. Hence, any restrictions (short or long-run) are over-identifying and can therefore be tested. This is achieved by estimating the model with and without restrictions on the $B$ matrix and comparing both log-likelihoods. In other words, an LR test is used and the test statistic has an asymptotic $\chi^{2}$ distribution with degrees of freedom equal to the number of restrictions.

\footnotetext{
${ }^{3}$ For instance, the LR test proceeds by restricting two diagonal elements of $\Lambda_{i}, i=2, \ldots, M$ to be equal and then comparing the log-likelihoods of the restricted and unrestricted models. This is done until all pairwise combinations of elements are exhausted. However, due to the highly nonlinear nature of the models, it is not uncommon for the EM algorithm to converge to the same parameter estimates and log-likelihood values for different pairwise combinations tests. In other words, when testing for the equality of pairwise $\Lambda_{i}, i=2, \ldots, M$ parameters, the EM algorithm could potentially converge to the same values over different pairwise tests, thereby giving the same results in each LR test. When working with trivariate models, three pairwise combinations exits, and it is usually found that the EM algorithm converges to the same optimum usually for two out of the three cases. Hence, two LR tests would have the same values. Therefore, Wald tests are deemed more reliable.
} 


\section{Model Results}

This section presents the main results of the paper in terms of testing the long-run identification schemes. Before discussing these results a brief word about the data used and the different models is in order.

\subsection{The Data and Model Specification}

Most data are from the Federal Reserve Economic Database (FRED). The dividends and earnings data are from Robert Schiller's webpage ${ }^{4}$ Hence, this analysis only investigates US data. In line with many of the papers mentioned in Table 1, the data is quarterly. The data range is from 1947:I - 2012:III, with the exception of dividends and earnings, which are until 2012:I. All variables are in real terms (except for the industrial production (IP) index) and in logs (except for the interest rate series). The interest rate is transformed to real terms by subtracting the CPI growth rate. Other variables are transformed to real terms by dividing by the percent level of the CPI. Figure 1 plots the data used along with recession periods according to NBER dating marked by the shaded bars.

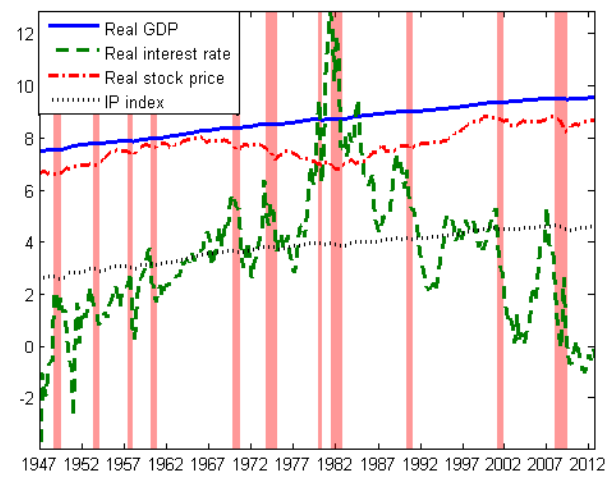

(a) GDP, r, s, IP

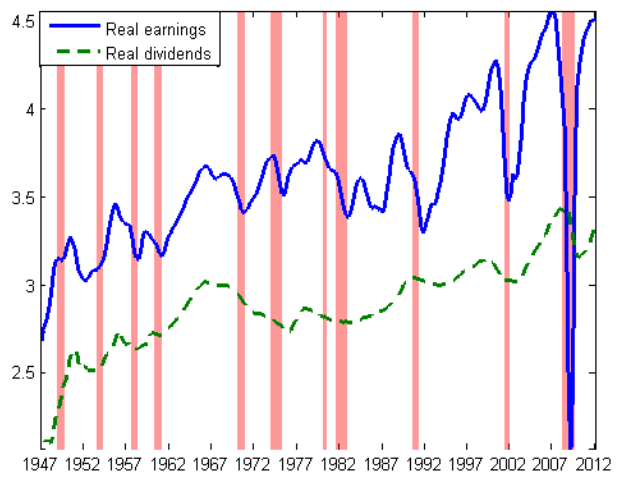

(b) e, d

Figure 1: Data used with recession dates indicated by the bars.

All variables are $I(1)$, meaning that they contain a unit root. This is according to Augmented Dickey-Fuller (ADF) and Kwiatkowski-Phillips-Schmidt-Shin (KPSS) tests. This is true even for the real interest rate series, although only at the $10 \%$ level according to the ADF test.

All popular models in Table 1 are investigated and are numbered as shown in Table 2 Both the Johansen (1995) trace test and the Saikkonen and Lütkepohl (2000) tests are used to

\footnotetext{
${ }^{4}$ Found at http://www.econ.yale.edu/ shiller/data.htm.
} 
test for cointegration in these models. According to these tests, only models IV and V show signs of cointegration and in each case the cointegrating rank, $r$ is 1 . Unit root and cointegration tests are carried out with the JMulTi software by Lütkepohl and Krätzig (2004).

Table 2: Summary of VAR and VEC models.

\begin{tabular}{l|c|l}
\hline \hline Model I & $y_{t}=\left[Y_{t}, r_{t}, s_{t}\right]^{\prime}$ & VAR in first differences \\
Model II & $y_{t}=\left[I P_{t}, r_{t}, s_{t}\right]^{\prime}$ & VAR in first differences \\
Model III & $y_{t}=\left[D_{t}, r_{t}, s_{t}\right]^{\prime}$ & VAR in first differences \\
Model IV & $y_{t}=\left[E_{t}, r_{t}, s_{t}\right]^{\prime}$ & VEC, $r=1$ \\
Model V & $y_{t}=\left[E_{t}, D_{t}, s_{t}\right]^{\prime}$ & VEC, $r=1$ \\
\hline \hline
\end{tabular}

\subsection{Model Restrictions}

As discussed, the structural models are all identified by means of restrictions on the long-run effects matrix. In particular, the type of restrictions on the SVAR models are all of the form as in 6). The restrictions on the SVEC models depend in part on the number of cointegrating relationships. In this case both models have one such relationship and hence no short-run restrictions are required, since $r(r-1) / 2=0$. Hence, the long-run restrictions as in (7) are used to identify the SVEC models. These identification methods provide three restrictions, as is necessary to just identify the models and are shown here again for convenience

$$
\Psi=\left[\begin{array}{ccc}
\star & 0 & 0 \\
\star & \star & 0 \\
\star & \star & \star
\end{array}\right] \quad \Xi B=\left[\begin{array}{ccc}
\star & 0 & 0 \\
\star & \star & 0 \\
\star & \star & 0
\end{array}\right]
$$

\subsection{MS Model Specification}

It is now necessary to specify the Markov switching (MS) models that are to be used. The number of lags and states can in principle be determined according to the model selection criteria developed by Psaradakis and Spagnolo 2006, who show that they work reasonably well. These criteria are the Akaike Information Criterion (AIC) and the Schwartz Criterion (SC). The AIC is calculated as $-2(\log$-likelihood $-n)$ and the SC is calculated as $-2 \log$-likelihood + $\log (T) n$, where $T$ is the sample size and $n$ is the number of free parameters of the model.

In principle the log-likelihood increases with the number of states used, although at a diminishing rate. When using too many states, however, there are usually convergence and 
estimation problems and ultimately it is not possible to escape the problem of too few observations for a given state. Therefore, these model selection criteria can provide reasonable judgement as to which model should be used since they penalize over-parameterized models. As already noted, these criteria can also help in selecting the number of model lags, however we prefer to choose model lag orders so as to avoid any residual autocorrelation. Hence, the optimal number of lags are determined by Portmanteau tests.

Table 3 shows results of the information criteria along with values of the log-likelihoods, $\ln (L)$ for all unrestricted models, i.e. models without any short or long-run restrictions. Minimum values of the information criteria are in bold. The maximum number of states considered is four. Beyond that no information criteria reaches a minimum and it becomes likely that there will be some states with very few observations. As noted, this causes convergence and estimation problems, often resulting in meaningless results.

Table 3: Information criteria of unrestricted models.

\begin{tabular}{|c|c|c|c|c|}
\hline Model & States & AIC & SC & $\ln (L)$ \\
\hline \multirow{4}{*}{ I: $y_{t}=\left[Y_{t}, r_{t}, s_{t}\right]^{\prime}$} & 1 & 1845.537 & -1738.716 & 952.768 \\
\hline & 2 & -1946.747 & -1822.123 & 1008.374 \\
\hline & 3 & -1961.999 & -1812.451 & 1023.000 \\
\hline & 4 & -1980.622 & -1798.028 & 1041.311 \\
\hline \multirow{4}{*}{ II: $y_{t}=\left[I P_{t}, r_{t}, s_{t}\right]^{\prime}$} & 1 & -1537.906 & -1367.364 & 816.953 \\
\hline & 2 & -1680.313 & -1492.006 & 893.157 \\
\hline & 3 & -1727.339 & -1514.161 & 923.669 \\
\hline & 4 & -1713.095 & -1467.941 & 925.547 \\
\hline \multirow{4}{*}{ III: $y_{t}=\left[D_{t}, r_{t}, s_{t}\right]^{\prime}$} & 1 & -1629.014 & -1490.600 & 853.507 \\
\hline & 2 & -1773.642 & -1617.482 & 930.821 \\
\hline & 3 & -1791.649 & -1610.646 & 946.825 \\
\hline & 4 & -1798.315 & -1585.371 & 959.157 \\
\hline \multirow{4}{*}{ IV: $y_{t}=\left[E_{t}, r_{t}, s_{t}\right]^{\prime}$} & 1 & -659.727 & -535.373 & 364.863 \\
\hline & 2 & -1220.152 & -1085.140 & 648.076 \\
\hline & 3 & -1297.577 & -1137.694 & 693.789 \\
\hline & 4 & -1327.046 & -1135.186 & 717.523 \\
\hline \multirow{4}{*}{$\mathrm{V}: y_{t}=\left[E_{t}, D_{t}, s_{t}\right]^{\prime}$} & 1 & -2775.382 & -2619.222 & 1431.691 \\
\hline & 2 & -3370.821 & -3193.367 & 1735.410 \\
\hline & 3 & -3455.565 & -3253.268 & 1784.783 \\
\hline & 4 & -3493.145 & -3258.906 & 1812.572 \\
\hline
\end{tabular}

Both information criteria agree for models II and V. For all other models the AIC favors more states than the SC. Unfortunately, convergence problems sometimes occur, even when using 4-state models. For instance, model III in four states turns out to have two states with 
very few observations in them. With model $V$ it is the case that the restricted 4-state model (i.e. the one with the long-run restrictions) fails to converge in the sense that the decomposition in (9) gives a singularity after a certain number of iterations. Given these considerations, two and three states are used for model III and three states are used for model V. For all other models the states suggested by the information criteria are taken into account.

It is also worth noting that models with one state, or simply SVAR and SVEC models, are not supported by any criterion. In particular, for the SVEC models IV and V the AIC and SC values are very high. Further, although not shown here, the log-likelihoods of models with a varying $B$ matrix over states are only slightly higher than those with a state invariant $B$ matrix; and the AIC and SC values are lower for a model with a state invariant $B$ matrix as opposed to a varying one. This means that the assumption of a state invariant $B$ matrix in (9) may well be justified, although this will formally be tested later on.

Portmanteau tests (not reported here) indicate that models I and III have no residual autocorrelation at 2 lags, models IV and $\mathrm{V}$ achieve this with 3 lags and model II needs 4 lags. Although, in fairness models IV and V still show signs of autocorrelation at the $10 \%$ level, which is also the case even when using higher lag orders. Therefore, in the interest of parsimony, for all models, we choose the lowest reasonable lag length possible.

\subsection{Estimation results}

For ease of presentation, the results of the MS models are presented according to number of states.

\subsubsection{2-state models}

Models I and III are best captured with two states according to the SC in Table 3 . The most relevant parameter estimates, along with standard errors and the covariance matrices (scaled by $10^{-3}$ ) of the 2 -state unrestricted 5 models, are shown in Table 4 . Both the values of the relative variance, $\lambda_{i j}, i=2, \ldots, M, j=1, \ldots, K$ parameters and the diagonal covariance matrix elements confirm that state 1 is the less volatile of the two states. This can also be seen from the smoothed probabilities of state 1 in Figure 2. In both models state 1 is never present during severe recessions, those with a greater than $2 \%$ contraction in GDP. For instance, the recession of 1958, the 1973-75 recession, both early 1980s recessions and the great recession of the late 2000s are captured by state 2 . 
Table 4: Parameter estimates, standard errors and covariance matrices (scaled by $10^{-3}$ ) for 2-state unrestricted models.

\begin{tabular}{|c|c|c|c|c|c|c|}
\hline Model & \multicolumn{3}{|c|}{ I: $y_{t}=\left[Y_{t}, r_{t}, s_{t}\right]^{\prime}$} & \multicolumn{3}{|c|}{ III: $y_{t}=\left[D_{t}, r_{t}, s_{t}\right]^{\prime}$} \\
\hline Parameter & estimate & \multicolumn{2}{|c|}{$\sigma$} & estimate & \multicolumn{2}{|c|}{$\sigma$} \\
\hline$\lambda_{21}$ & 4.295 & \multicolumn{2}{|c|}{1.088} & 2.398 & \multicolumn{2}{|c|}{0.824} \\
\hline$\lambda_{22}$ & 2.523 & \multicolumn{2}{|c|}{0.706} & 10.004 & \multicolumn{2}{|c|}{2.531} \\
\hline$\lambda_{23}$ & 8.084 & \multicolumn{2}{|c|}{1.991} & 6.777 & \multicolumn{2}{|c|}{1.730} \\
\hline$p_{11}$ & 0.945 & \multicolumn{2}{|c|}{0.021} & 0.939 & \multicolumn{2}{|c|}{0.026} \\
\hline$p_{22}$ & 0.775 & \multicolumn{2}{|c|}{0.081} & 0.735 & \multicolumn{2}{|c|}{0.086} \\
\hline \multirow{3}{*}{$\Sigma(1)$} & 0.045 & - & - & 0.067 & - & - \\
\hline & 0.893 & 278.148 & - & -0.110 & 227.717 & - \\
\hline & 0.033 & -0.082 & 2.288 & 0.033 & -1.199 & 2.440 \\
\hline \multirow{3}{*}{$\Sigma(2)$} & 0.195 & - & - & 0.559 & - & - \\
\hline & 1.819 & 2065.566 & - & -7.257 & 1913.660 & - \\
\hline & 0.296 & -12.278 & 6.423 & -0.029 & -11.653 & 5.973 \\
\hline
\end{tabular}



(a) Model I

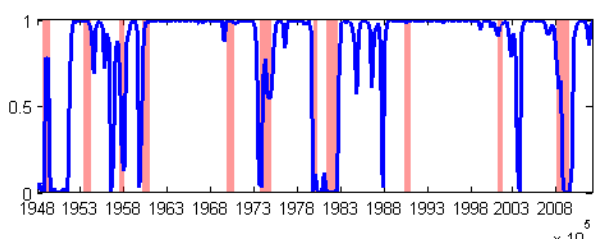

(b) Model III

Figure 2: Smoothed probabilities of state 1 along with recession dates.

\subsubsection{3-state models}

A 3-state model is the most prevalent one. In particular, given the convergence and interpretation issues mentioned above, three states are used instead of four for some of the models. Hence, models II, III, IV and V are considered in three MS volatility states. As with the 2-state models, the relevant parameter estimates along with standard errors are shown in Table 5 and the smoothed probabilities of the states are shown in Figure 3 .

Table 5 shows that the $\lambda_{i j}, i=2, \ldots, M, j=1, \ldots, K$ parameters still seem quite diverse,

\footnotetext{
${ }^{5}$ Here unrestricted refers to no short or long-run restrictions on the state invariant $B$ matrix.

${ }^{6}$ Note that when using more than 2 states, all smoothed probabilities need to be displayed since it is no longer the case that one is the mirror image of the other.
} 






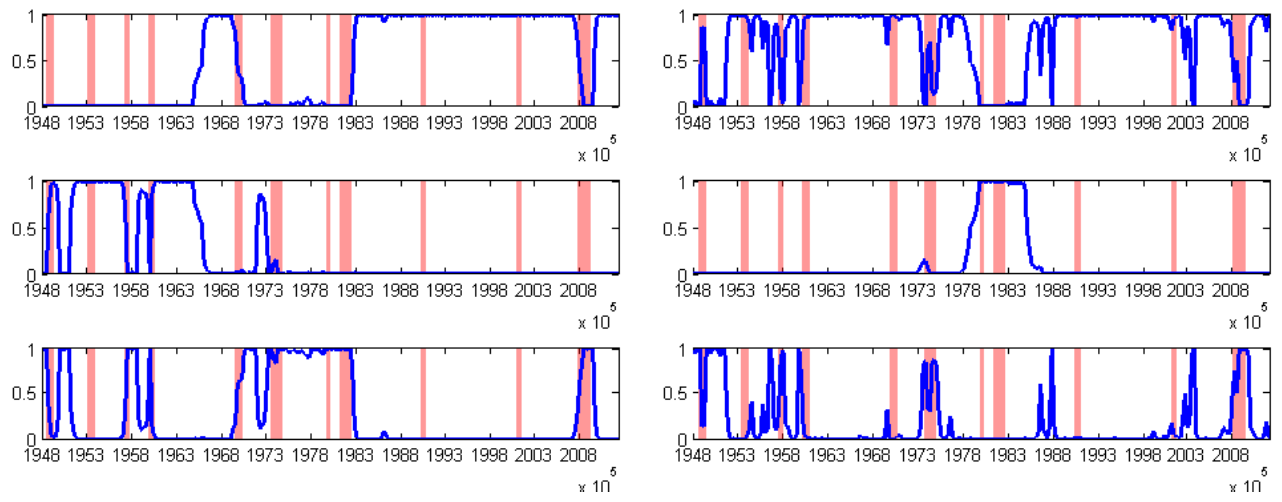

(a) Model II

(b) Model III
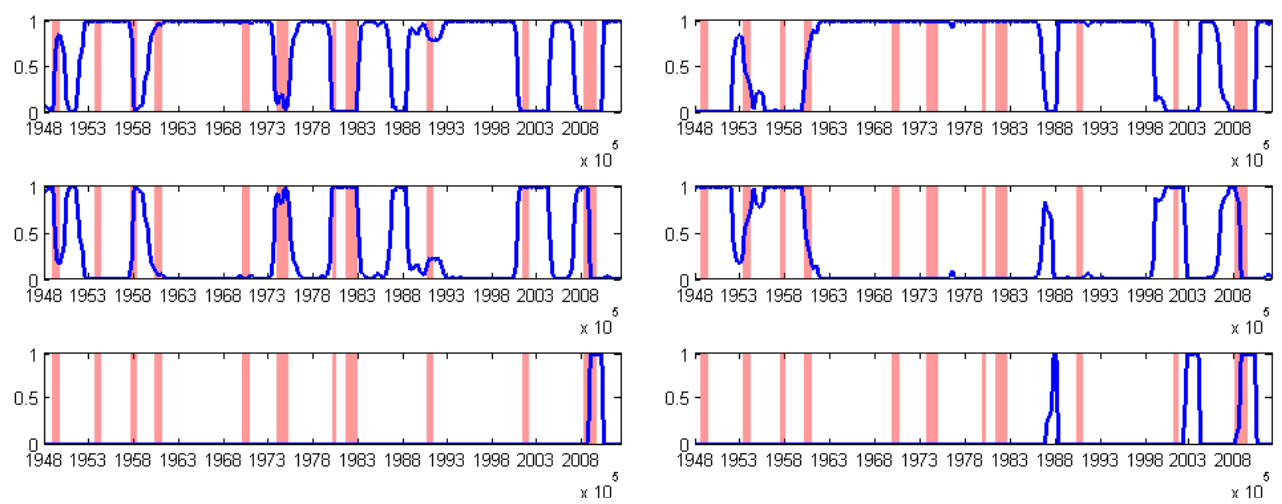

(c) Model IV

(d) Model V

Figure 3: Smoothed probabilities of State 1 (top), State 2 (middle) and State 3 (bottom) along with recession dates.

however in some cases their standard errors are also high. Also worth noting is that some of these parameters have rather low values, below 1, meaning that the relative variance in the given state is less than that of the first state. This can also be observed by the diagonal elements of the covariance matrices at the bottom part of the table. With the exception of model IV, the variances are not always increasing with a given state. This means that interpretation of the states is slightly more complex than with the 2-state models above.

From the smoothed probabilities in Figure 3 it can be seen that for all models, state 1 is not usually associated with severe recessions. In particular, this is especially the case for model IV and, to a lesser extent for model V. State 2 largely tends to capture recession periods along with some time interval around them. For model III, state 2 is only associated with the early 1980s, where notably Binswanger (2004a|b|c), Groenewold (2004) and Jean and Eldomiaty (2010) all argue for the existence of a structural break in the relationship between stock prices 
and their fundamentals around that time period. However, no other model indicates any significant event around that time to warrant its own state. The great recession is always given by the third state. In model IV this is also the only occurrence of that state and in the other models state 3 is usually associated with severe recession periods. Hence, it can be interpreted as being the most volatile state.

The estimates of the transition probabilities in Table 5 are usually close to one, with the slight exception of $p_{33}$. 7 This means that the states tend to be quite persistent as seen in Figure 3 in that the smoothed probabilities do not fluctuate often. The lower persistence of the third state is also something we would expect, since it is usually the case that crisis periods tend to be more transitory than economically stable periods. In this case the duration of the third state is roughly between 3 and 8 quarters, depending on the model used. This is a reasonable recession duration estimate given the data range we are using.

Finally, as noted in section 3, when using three or more Markov states, the assumption of a state invariant $B$ matrix can be tested. The test distribution is asymptotically $\chi^{2}$ with $(1 / 2) M K(K+1)-K^{2}-(M-1) K$ degrees of freedom, or in this case 3. The $p$-values of such a test are shown in the bottom part of Table 5. Clearly, at conventional critical levels the null hypothesis of a state invariant $B$ matrix cannot be rejected. Hence, one of the necessary model assumptions is justified by the data.

\subsubsection{4-state models}

Models I and IV are also considered in 4 states. Results of their parameter estimates and smoothed probabilities are displayed in Table 6 and Figure 4 respectively. These models have the most parameters out of all the models considered thus far, which consequently makes it more complicated to classify their states. The diagonal elements of the covariance matrices in the lower half of Table 6 do not always increase with the given state. Looking at the smoothed probabilities, it is however possible to classify state 1 as the least volatile state since it tends to avoid most recession periods. State 4 on the other hand tends to capture periods of severe recessions and for model IV only the great recession is present in that state. Hence, state 4 can be considered as the one with the highest volatility. States 2 and 3 are similar especially for model IV in that they are present during different recession periods.

It is again worth noting that the point of a structural break in the early 1980s can be somewhat justified when looking at the smoothed probabilities of state 1 for model I in Panel (a) of Figure 4 . This state seems to be present mainly after the early 1980s, which could indicate a change in some fundamental relationship due to the lower volatility after that period. Also

\footnotetext{
${ }^{7}$ These are not the only unrestricted elements of the transition probabilities, however to save space only these ones are displayed as they tend to be of most interest.
} 
Table 6: Parameter estimates, standard errors and covariance matrices (scaled by $10^{-3}$ ) for 4 -state unrestricted models. Tests for a state-invariant $B$ matrix at the bottom.

\begin{tabular}{|c|c|c|c|c|c|c|}
\hline Model & \multicolumn{3}{|c|}{$\mathrm{I}: y_{t}=\left[Y_{t}, r_{t}, s_{t}\right]^{\prime}$} & \multicolumn{3}{|c|}{$\mathrm{IV}: y_{t}=\left[E_{t}, r_{t}, s_{t}\right]^{\prime}$} \\
\hline Parameter & estimate & \multicolumn{2}{|c|}{$\sigma$} & estimate & \multicolumn{2}{|c|}{$\sigma$} \\
\hline$\lambda_{21}$ & 3.734 & \multicolumn{2}{|c|}{1.051} & 1.168 & \multicolumn{2}{|c|}{0.463} \\
\hline$\lambda_{22}$ & 0.385 & \multicolumn{2}{|c|}{0.092} & 9.553 & \multicolumn{2}{|c|}{2.488} \\
\hline$\lambda_{23}$ & 0.946 & \multicolumn{2}{|c|}{0.411} & 1.116 & \multicolumn{2}{|c|}{0.370} \\
\hline$\lambda_{31}$ & 2.465 & \multicolumn{2}{|c|}{1.049} & 9.706 & \multicolumn{2}{|c|}{2.714} \\
\hline$\lambda_{32}$ & 0.397 & \multicolumn{2}{|c|}{0.196} & 1.250 & \multicolumn{2}{|c|}{0.399} \\
\hline$\lambda_{33}$ & 5.685 & \multicolumn{2}{|c|}{1.835} & 2.776 & \multicolumn{2}{|c|}{0.798} \\
\hline$\lambda_{41}$ & 9.883 & \multicolumn{2}{|c|}{3.521} & 1086.648 & \multicolumn{2}{|c|}{651.861} \\
\hline$\lambda_{42}$ & 6.081 & \multicolumn{2}{|c|}{1.916} & 49.092 & \multicolumn{2}{|c|}{34.599} \\
\hline$\lambda_{43}$ & 1.763 & \multicolumn{2}{|c|}{0.798} & 1.544 & \multicolumn{2}{|c|}{0.975} \\
\hline$p_{11}$ & 0.931 & \multicolumn{2}{|c|}{0.097} & 0.948 & \multicolumn{2}{|c|}{0.027} \\
\hline$p_{22}$ & 0.948 & \multicolumn{2}{|c|}{0.040} & 0.793 & \multicolumn{2}{|c|}{0.090} \\
\hline$p_{33}$ & 0.668 & \multicolumn{2}{|c|}{0.169} & 0.816 & \multicolumn{2}{|c|}{0.068} \\
\hline$p_{44}$ & 0.795 & \multicolumn{2}{|c|}{0.082} & 0.828 & \multicolumn{2}{|c|}{0.362} \\
\hline \multirow{3}{*}{$\Sigma(1)$} & 0.022 & - & - & 0.305 & - & - \\
\hline & 0.254 & 362.760 & - & -0.171 & 217.329 & - \\
\hline & 0.039 & -1.357 & 2.281 & 0.117 & -1.879 & 2.164 \\
\hline & 0.077 & - & - & 0.358 & - & - \\
\hline$\Sigma(2)$ & 1.430 & 180.694 & - & -1.881 & 2073.731 & - \\
\hline & 0.062 & -0.359 & 2.312 & 0.141 & -11.461 & 2.463 \\
\hline & 0.214 & - & - & 2.938 & - & - \\
\hline$\Sigma(3)$ & 3.287 & 2148.423 & - & 0.236 & 272.111 & - \\
\hline & 0.118 & -4.495 & 2.259 & 0.580 & -3.471 & 6.030 \\
\hline & 0.082 & - & - & 327.892 & - & - \\
\hline$\Sigma(4)$ & 0.975 & 1261.377 & - & 53.393 & 10667.553 & - \\
\hline & 0.265 & -7.034 & 17.680 & 40.652 & -47.875 & 8.602 \\
\hline & & $H_{0}$ & ate in & nt $B$ & & \\
\hline$p$-value & & 0.375 & & & 0.082 & \\
\hline
\end{tabular}



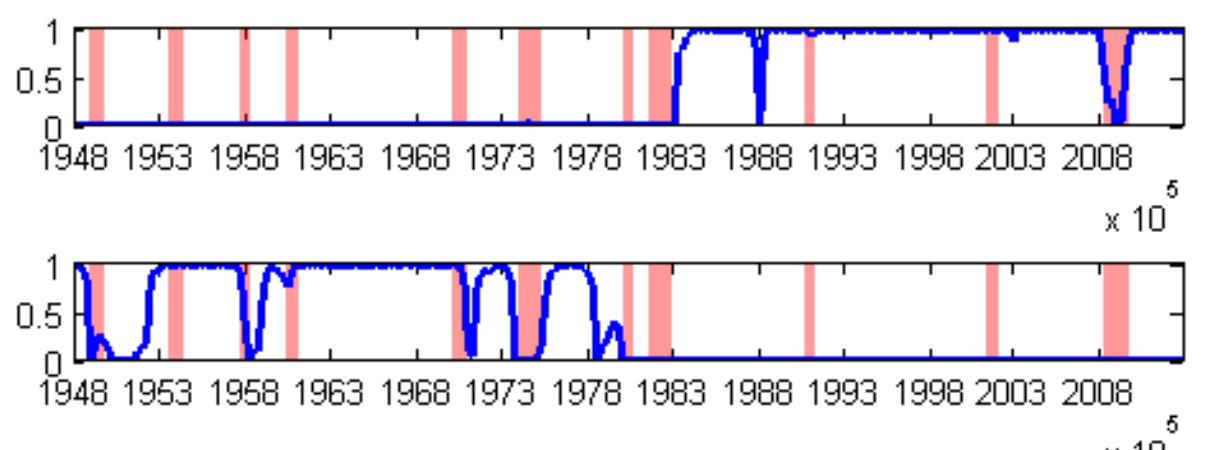

$\times 10^{5}$
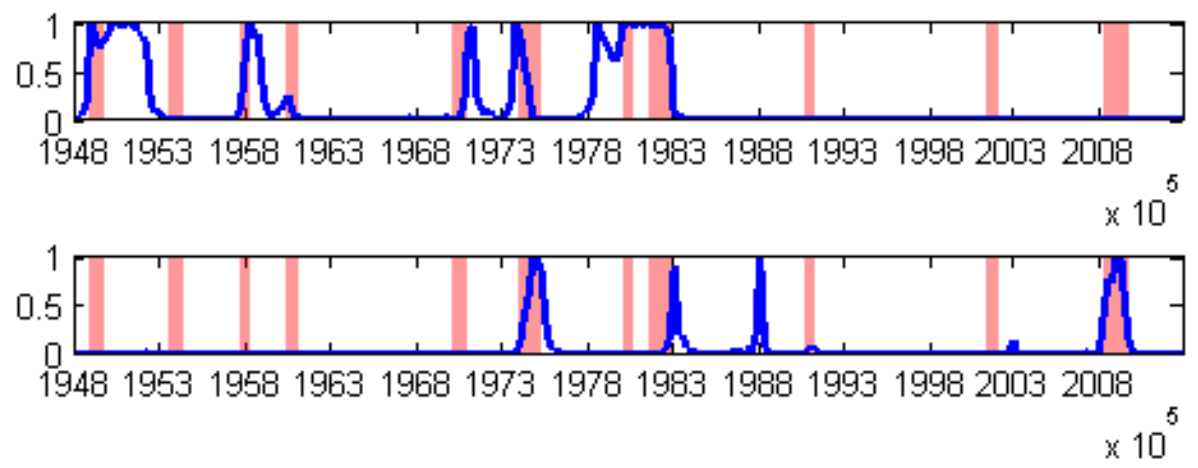

(a) Model I


$\times 10^{5}$

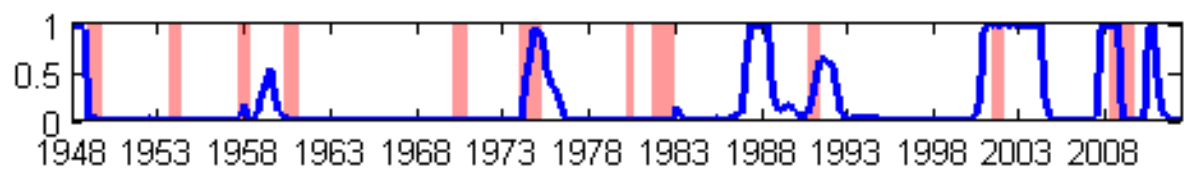

$\times 10^{5}$

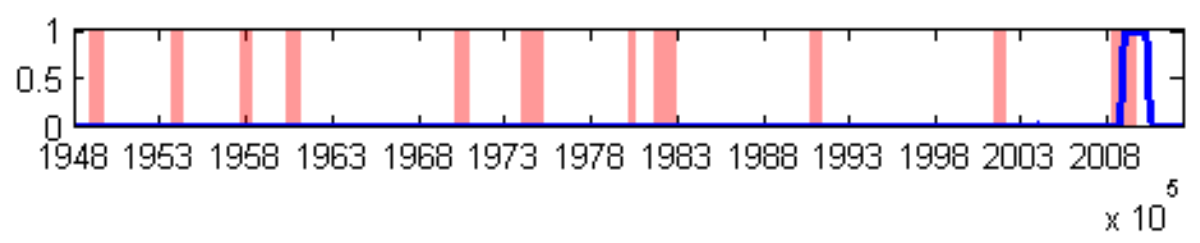

(b) Model IV

Figure 4: Smoothed probabilities of state 1 to state 4 (from top to bottom) along with recession dates. 
interesting is that both in 3 and 4 states, the most volatile state of model IV only captures the great recession and nothing else. This may not be that surprising however - when looking at the real earnings series in Panel (b) of Figure 1 a huge swing in real earnings is observed during the period of the financial crisis. Whether this particular period drives the results is investigated in the next section on model robustness.

Finally, as with the 3-state models, the assumption of a state invariant $B$ matrix is formally tested. The resulting $p$-values are displayed at the bottom of Table 8 . At the $5 \%$ critical level the hypothesis of a state invariant $B$ matrix cannot be rejected. This means that for both three and four-state models, this assumption is supported by the data.

\subsection{Testing the Identification Restrictions}

We now turn to testing whether the restrictions in (6) and (7) are supported by the data. The first step is by testing whether the state invariant $B$ matrix is identified through heteroskedasticity.

\subsubsection{Testing for distinct lambda parameters}

As discussed in section 3, in order for the $B$ matrix to be identified through heteroskedasticity, it is necessary that all pairwise $\lambda_{i j}, i=2, \ldots, M, j=1, \ldots, K$ elements be distinct at least once in any $\Lambda_{i}, i=2, \ldots, M$ matrix. Since the standard errors are available, this is most easily tested by means of a Wald test. Likelihood ratio (LR) tests are also used, however, as already noted, such tests can suffer from convergence problems and in most cases converge to the same values for different hypotheses at least once. Nevertheless, when comparable, both tests yield the same results. The hypotheses and $p$-values of the Wald tests by models are given in Table 7.

The test statistic follows a $\chi^{2}$ distribution with degrees of freedom equal to the number of joint hypotheses being examined. Taking a $5 \%$ or even a $10 \%$ critical level, no 2 -state model can reject the null of at least one parameter pair of diagonal $\Lambda_{2}$ parameters being equal. For the 3-state models this is only true for model IV and all null hypotheses are rejected for the 4-state models.

It is promising that for all models the null hypotheses are rejected for a given number of states. In particular, the null hypotheses for models I, III and IV are all rejected when using a higher number of states. This means that for all models the $B$ matrix is uniquely identified through heteroskedasticity. Hence, any restrictions now on that matrix become over-identifying and are in a position to be tested.

\footnotetext{
${ }^{8}$ The test statistic is asymptotically $\chi^{2}$ distributed with 6 degrees of freedom.
} 
Table 7: Null hypotheses by state and $p$-values of Wald tests for all models.

\begin{tabular}{l|l|ccc}
\hline States & Model & \multicolumn{3}{c}{$p$-values } \\
\hline \hline \multirow{3}{*}{2} & $H_{0}$ & $\lambda_{21}=\lambda_{22}$ & $\lambda_{21}=\lambda_{23}$ & $\lambda_{22}=\lambda_{23}$ \\
\cline { 2 - 5 } & I: $\left[Y_{t}, r_{t}, s_{t}\right]^{\prime}$ & 0.180 & 0.091 & 0.008 \\
& III: $\left[D_{t}, r_{t}, s_{t}\right]^{\prime}$ & 0.007 & 0.031 & 0.266 \\
\hline \multirow{4}{*}{3} & $H_{0}$ & $\lambda_{21}=\lambda_{22}, \lambda_{31}=\lambda_{32}$ & $\lambda_{21}=\lambda_{23}, \lambda_{31}=\lambda_{33}$ & $\lambda_{22}=\lambda_{23}, \lambda_{32}=\lambda_{33}$ \\
\cline { 2 - 5 } & II: $\left[I P_{t}, r_{t}, s_{t}\right]^{\prime}$ & 0.001 & 0.000 & 0.000 \\
& III: $\left[D_{t}, r_{t}, s_{t}\right]^{\prime}$ & 0.019 & 0.009 & 0.005 \\
& IV: $\left[E_{t}, r_{t}, s_{t}\right]^{\prime}$ & 0.263 & 0.014 & 0.016 \\
& V: $\left[E_{t}, D_{t}, s_{t}\right]^{\prime}$ & 0.001 & 0.000 & 0.000 \\
\hline \multirow{4}{*}{4} & $H_{0}$ & $\lambda_{21}=\lambda_{22}, \lambda_{31}=\lambda_{32}, \lambda_{41}=\lambda_{42}$ & $\lambda_{21}=\lambda_{23}, \lambda_{31}=\lambda_{33}, \lambda_{41}=\lambda_{43}$ & $\lambda_{22}=\lambda_{23}, \lambda_{32}=\lambda_{33}, \lambda_{42}=\lambda_{43}$ \\
\cline { 2 - 5 } & I: $\left[Y_{t}, r_{t}, s_{t}\right]^{\prime}$ & 0.001 & 0.000 & 0.001 \\
& IV: $\left[E_{t}, r_{t}, s_{t}\right]^{\prime}$ & 0.000 & 0.034 & 0.000 \\
\hline \hline
\end{tabular}

\subsubsection{Testing the restrictions}

The restrictions to be tested are the lower triangular long-run identification restrictions mainly used in the literature, given by (6) and (7) for SVAR and SVEC models respectively. These restrictions are tested by comparing the log-likelihood values of the unrestricted (and identified) models with the restricted models according to (6) and (7) by means of an LR test. The results of these tests are given in Table 8 . The distribution of the test statistic is asymptotically $\chi^{2}$ with 3 degrees of freedom since all restricted models have 3 restrictions so that they are just-identified in the traditional sense. The alternative hypothesis is the model without any restrictions on the state invariant $B$ matrix.

Starting with the 2-state models, the long-run restrictions for model I are accepted at the $10 \%$ critical value. However, that model did not have a uniquely identified $B$ matrix as indicated in Table 7. Hence, any conclusions on the acceptance of the identification scheme become somewhat ambiguous. Moving on to the 3 -state models, at a $5 \%$ critical value the long-run restrictions for model III are accepted. These restrictions are resoundingly rejected for all other 3-state models, given that their $p$-values are very close to zero. Finally, in four volatility states, the long-run restrictions for model I are again accepted, this time at the $5 \%$ critical level. Now however, the $B$ matrix is identified through heteroskedasticity, hence this result indeed shows support for the long-run identification scheme.

We therefore conclude that only models I and III in four and three states respectively have support from the data for the lower triangular long-run identification scheme. Such restrictions could indeed categorize shocks as fundamental and non-fundamental as the literature tends to do. With other models these restrictions do not seem to be warranted by the data, 
Table 8: $p$-values for LR tests of the long-run restrictions. The alternative hypothesis is a state invariant, unrestricted $B$ matrix.

\begin{tabular}{|c|c|c|c|c|}
\hline & model & $H_{0}$ & LR test & $p$-value \\
\hline \multirow[t]{2}{*}{2 states } & $\mathrm{I}:\left[Y_{t}, r_{t}, s_{t}\right]^{\prime}$ & 6 & 0.698 & 0.874 \\
\hline & III: $\left[D_{t}, r_{t}, s_{t}\right]^{\prime}$ & 6 & 8.735 & 0.033 \\
\hline \multirow[t]{4}{*}{3 states } & II: $\left[I P_{t}, r_{t}, s_{t}\right]^{\prime}$ & 6 & 56.084 & $4.031 \times 10^{-12}$ \\
\hline & III: $\left[D_{t}, r_{t}, s_{t}\right]^{\prime}$ & 6 & 7.581 & 0.056 \\
\hline & $\mathrm{IV}:\left[E_{t}, r_{t}, s_{t}\right]^{\prime}$ & (7) & 19.764 & $1.900 \times 10^{-4}$ \\
\hline & $\mathrm{V}:\left[E_{t}, D_{t}, s_{t}\right]^{\prime}$ & 7 & 25.416 & $1.264 \times 10^{-5}$ \\
\hline \multirow[t]{2}{*}{4 states } & I: $\left[Y_{t}, r_{t}, s_{t}\right]^{\prime}$ & 6 & 6.601 & 0.086 \\
\hline & $\mathrm{IV}:\left[E_{t}, r_{t}, s_{t}\right]^{\prime}$ & 7 & 72.808 & $1.110 \times 10^{-15}$ \\
\hline
\end{tabular}

meaning that the identified shocks can probably not be interpreted as fundamental and nonfundamental.

Finally, it is worth mentioning that in most of the literature VAR models instead of VEC models are used. However, both cointegration tests indicate a strong presence of cointegration in models IV and V. Therefore, it would be more advisable to use the VEC form for such models. Note that a VAR in levels form is also possible, however this would again diverge from the literature, which mainly uses VARs in first differences.

\section{Robustness Analysis}

This section investigates whether the results obtained thus far rely to some extent on the exact model specifications used. Table 8 shows that the number of states do not seem to influence the final results. They only seem to matter for identifying the $B$ matrix in (9) up to changes in sign and column ordering. A similar conclusion (although not reported here) can be drawn for the number of lags; though models with different lag orders may have residual autocorrelation as indicated by Portmanteau tests, the results in Table 8 stay similar depending on the critical level threshold chosen to evaluate them.

In order to investigate model robustness, it would be more relevant for example to try to determine whether the sample range could somehow drive the results obtained thus far. For instance, the smoothed probabilities of model IV in panels (c) and (b) of Figures 3 and 4 respectively show that there is one state that always captures the financial crisis. It would be 
interesting to investigate what would happen if the sample is cut to exclude the crisis years. Further, some papers for instance, Binswanger (2000), Binswanger 2004b) and Jean and Eldomiaty (2010) use data starting from 1953 to avoid having the Korean War influence their results. Hence, removing the turbulent beginning and end of the sample would give a good indication of how robust the results are. Therefore, we only keep the observations from 1953:I - 2007:III for the robustness analysis.

Another factor potentially influencing the results could be the Markov switching (MS) specification itself. For example, as was already clarified, a MS model in heteroskedasticity needs to be used so that the $B$ matrix can be identified and any restrictions on it can be tested. There is, however, little reason to assume that no other parameters can switch. When using data such as interest rates and stock price indices, it may well be the case that the intercept term is also subject to the same Markov regime switches as the covariance matrix. Indeed, stock prices tend to rise (fall) in periods of low (high) volatility. Allowing the intercept term to switch is another way of testing in how far the results obtained above are robust. Note that the autoregressive parameters could potentially also be switching, however the case for them to switch is harder to justify and to interpret. Further, switching autoregressive parameters may cause estimation issues; in that the number of parameters to be estimated increases and the data range may be too limited to give accurate estimates of all these parameters when using many MS states. Hence, we decide to investigate a model only with a further switching intercept term in addition to the switching covariance matrix. The VAR model, (1) then looks as follows

$$
y_{t}=v\left(S_{t}\right)+A_{1} y_{t-1}+A_{2} y_{t-2}+\ldots+A_{p} y_{t-p}+u_{t},
$$

where $S_{t}$ follows a discrete valued first order Markov process as before and $u_{t}$ still has the same distributional assumption as in (8). The reduced form VEC model is similar to (10) with the switching intercept being $v_{0}\left(S_{t}\right)$.

Finally, the Dow Jones index is not the only index followed by market participants. It consists of only 30 companies, whereas for example, the S\&P 500 index consists of 500 companies as its name suggests. Even though these indices are closely correlated and one may argue that any idiosyncracies are diversified away, the choice of index may still influence the earlier conclusions. Further, data on the S\&P 500 starts from 1957:I thereby giving a joint robustness check in terms of a different stock price index and data range compared with the original analysis.

It is worth noting that by reducing the sample range or introducing a new stock price index, the models need to again be tested for cointegration. It turns out that the cointegration relationships discovered earlier are all kept. 9 Hence, models IV and V are still of the VEC

\footnotetext{
${ }^{9}$ Only model IV with the 1953:1 - 2007:III sample range shows weak signs of cointegration, however, it is still
} 
form, with a cointegrating rank of 1, while models I - III are still of the VAR form. This is reassuring since cointegration is assumed to involve long-run relationships, which should not be expected to change due to changes in the sample range. Further, for all robustness specifications, the same lag lengths as in the original analysis are kept. This could in principle lead to some residual autocorrelation, however by using the same lag lengths the results from the robustness checks can best be compared to the original ones.

Table 9: $p$-values for tests of a state invariant $B$ matrix.

\begin{tabular}{l|l|ccc||c}
\multicolumn{7}{c}{$H_{0}$ : state invariant $B$} \\
& model & $\begin{array}{c}\text { 1953:I - } \\
\text { 2007:III }\end{array}$ & intercept & S\&P 500 & Original \\
\hline \hline \multirow{3}{*}{3 states } & II: $\left[I P_{t}, r_{t}, s_{t}\right]^{\prime}$ & $1.332 \times 10^{-9}$ & 0.138 & 0.419 & 0.150 \\
& III: $\left[D_{t}, r_{t}, s_{t}\right]^{\prime}$ & 0.191 & 0.106 & 0.051 & 0.204 \\
& IV: $\left[E_{t}, r_{t}, s_{t}\right]^{\prime}$ & 0.008 & 0.256 & 0.307 & 0.600 \\
& V: $\left[E_{t}, D_{t}, s_{t}\right]^{\prime}$ & $1.459 \times 10^{-5}$ & 0.901 & 0.006 & 0.946 \\
\hline \multirow{2}{*}{4 states } & $\mathrm{I}:\left[Y_{t}, r_{t}, s_{t}\right]^{\prime}$ & $3.195 \times 10^{-8}$ & 0.611 & $2.679 \times 10^{-6}$ & 0.375 \\
& $\mathrm{IV}:\left[E_{t}, r_{t}, s_{t}\right]^{\prime}$ & $3.840 \times 10^{-5}$ & 0.481 & 0.999 & 0.082 \\
\hline \hline
\end{tabular}

As in the original analysis, it is first necessary to confirm whether the assumption of a state invariant $B$ matrix is justified. Recall, that this assumption can be tested for models with three or more Markov states. The test distribution is given as before; asymptotically $\chi^{2}$ with $(1 / 2) M K(K+1)-K^{2}-(M-1) K$ degrees of freedom. The $p$-values of such tests for all three robustness specifications are given in Table 9 . The most right column of the table shows the original $p$-values from Tables 5 and 6 for comparison. It can be seen that the null hypothesis is usually accepted at the $5 \%$ significance level. A notable exception to this is for the shortened sample range. There, in most cases very low $p$-values are obtained, meaning a rejection of the assumption of a state invariant $B$ matrix. Overall however, from the results of the other robustness specifications, we can conclude that the assumption of a state invariant $B$ matrix is a rather robust one.

Assuming that there indeed is enough justification for a state invariant $B$ matrix, we then test whether this matrix is identified through heteroskedasticiy and if so whether the longrun restrictions in (6) and (7) are supported by the data. The $p$-values for tests of the long-run restrictions are reported in Table $10 \|^{0}$. The most right column of the table again shows the present.

${ }^{10}$ The test distributions are again asymptotically $\chi^{2}$ with three degrees of freedom, since both identification 
Table 10: $p$-values for LR tests of the long-run restrictions for different robustness specifications. The alternative hypothesis is a state invariant, unrestricted $B$ matrix.

\begin{tabular}{|c|c|c|c|c|c|c|}
\hline & model & $H_{0}$ & $\begin{array}{l}\text { 1953:I - } \\
\text { 2007:III }\end{array}$ & intercept & S\&P 500 & Original \\
\hline \multirow[t]{2}{*}{2 states } & I: $\left[Y_{t}, r_{t}, s_{t}\right]^{\prime}$ & (6) & 0.911 & 0.644 & 0.699 & 0.874 \\
\hline & III: $\left[D_{t}, r_{t}, s_{t}\right]^{\prime}$ & (6) & 0.287 & 0.032 & 0.888 & 0.033 \\
\hline \multirow[t]{4}{*}{3 states } & II: $\left[I P_{t}, r_{t}, s_{t}\right]^{\prime}$ & (6) & $1.332 \times 10^{-9^{*}}$ & $2.854 \times 10^{-10^{*}}$ & $0.038^{*}$ & $4.031 \times 10^{-12^{*}}$ \\
\hline & III: $\left[D_{t}, r_{t}, s_{t}\right]^{\prime}$ & 6 & $0.272^{*}$ & 0.474 & $0.984^{*}$ & $0.056^{*}$ \\
\hline & $\mathrm{IV}:\left[E_{t}, r_{t}, s_{t}\right]^{\prime}$ & (7) & $0.277^{*}$ & $2.718 \times 10^{-4}$ & $1.373 \times 10^{-4}$ & $1.900 \times 10^{-4}$ \\
\hline & $\mathrm{V}:\left[E_{t}, D_{t}, s_{t}\right]^{\prime}$ & (7) & $1.325 \times 10^{-10^{*}}$ & $7.704 \times 10^{-13^{*}}$ & $0.055^{*}$ & $1.264 \times 10^{-5^{*}}$ \\
\hline \multirow[t]{2}{*}{4 states } & I: $\left[Y_{t}, r_{t}, s_{t}\right]^{\prime}$ & 6 & 0.451 & $0.023^{*}$ & $0.026^{*}$ & $0.081^{*}$ \\
\hline & $\mathrm{IV}:\left[E_{t}, r_{t}, s_{t}\right]^{\prime}$ & (7) & $0.002^{*}$ & $9.525 \times 10^{-9^{*}}$ & $6.387 \times 10^{-7^{*}}$ & $1.110 \times 10^{-15^{*}}$ \\
\hline
\end{tabular}

* The $B$ matrix is identified up to changes in sign.

original $p$-values from Table 8 for comparison. The stars in the table indicate when the $B$ matrix is identified (through heteroskedasticity) up to changes in sign and column ordering, i.e. when the null hypotheses in Table 7 are rejected.

Analyzing the results by model, it can be seen that there is no new conclusion for model I in two states; in all cases, the $B$ matrix is not identified and the restricted model is accepted. When using a $1 \%$ critical value, the original conclusion also holds for that model in four states. Granted, the $p$-values are usually less than in the original specification, however they are not arbitrarily close to zero, as is the case for some of the other models. Only for the short sample range is the $B$ matrix not identified through heteroskedasticity, making any conclusion on accepting the identification scheme ambiguous.

Moving on to model II, its identification scheme is rejected over all robustness specifications, except when using the S\&P 500 series and a $1 \%$ critical value. Overall, these results suggest that the identification restrictions for model II can largely be rejected. This means that when using industrial production data instead of GDP data the structural shocks may not be properly identified in the sense that the structural identifying restrictions are not supported by the data. This could be due to the stock price index and interest rates being more reflective of GDP rather than the industrial production index. It illustrates the need of being able to test a given identification scheme so as to let the data speak up about the restrictions. schemes contain three restrictions. 
This indicates that simply using the same identification scheme for different although closely related models, does not necessarily lead to the identification of the same type of shocks.

The original conclusion for model III in two states does not change since again the $B$ matrix is not identified for any of the models. When using three states, the restricted model is accepted, as was originally the case. The $p$-values are in all cases higher than for the original model. However, the $B$ matrix is not identified through heteroskedasticity when using a model with a switching intercept term. Nevertheless, it can be concluded with reasonable confidence that the identification scheme in (6) is robustly accepted for model III.

For model IV, the original conclusion is widely upheld except when using a shorter sample range and three states. Then the identification restrictions in (7) are accepted. This could mainly be due to omission of the financial crisis period. As seen in Figure 1, panel (b), real earnings were severely affected during that time period. However, since the original conclusion rejecting the long-run identification scheme holds in most cases, it can be said to be quite robust.

Similarly for model $\mathrm{V}$, the original conclusion is only rejected in one instance at the $5 \%$ critical level. Overall, the results in Table 10 lend some credibility to the original findings and show that they are rather robust over different model specifications.

To complete this analysis, a brief note on the smoothed probabilities is in order. For the 2state models, the periods depicted by the smoothed probabilities are very similar to the ones shown in Figure 2. In other words, they are not really different from the original ones.

The smoothed probabilities of the 3-state MS-SVAR models do not seem as robust to model specification however. In particular, for model II a similar picture as that in Panel
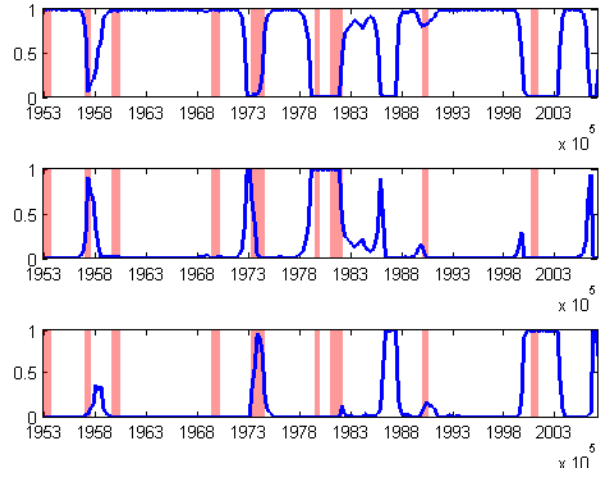

(a) Model IV

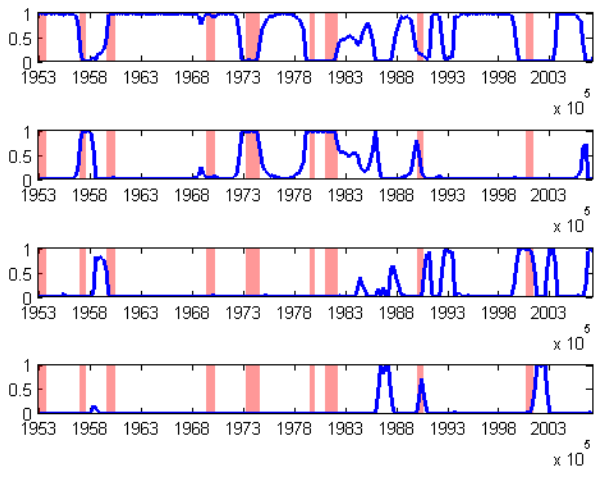

(b) Model IV

Figure 5: Smoothed probabilities of model IV with the 1953:I - 2007:III sample range with 3, (a) and 4, (b) states. 
(a) of Figure 3 is only obtained when using a model with a switching intercept term. Similarly, for model III, none of the robustness specifications show a unique event in the early 1980s as is the case in Panel (b) of Figure 3. Nevertheless, for all robustness models, the first state still depicts the most stable periods and recessions and crises are captured by states two and three as before.

The MS-SVEC models, on the other hand, tend to deliver rather robust smoothed probabilities over different robustness specifications when modeled in three Markov states. They largely resemble the ones in Panels (c) and (d) of Figure 3. Interestingly, when cutting the sample to before the financial crisis, state three no longer indicates a unique event for model IV. Rather recession periods are depicted by both states two and three as can be seen in Panel (a) of Figure 5. Model $\mathrm{V}$ with a switching intercept displays the financial crisis as a unique event in the third state.

A similar conclusion can be made for the 4-state models in the sense that the smoothed probabilities of the MS-SVEC models are more robust than those of the MS-SVAR models. It is also the case with these models that the first state depicts the more stable economic periods, while other states capture more turbulent times. As in the 3-state case, when excluding the financial crisis, the fourth state no longer depicts a unique event for model IV, as shown in Figure 5, (b).

The robustness tests show that the results obtained earlier are not merely subject to chance and that there is some credible evidence either in favor or against the relevant identification scheme of a given model.

\section{Conclusion}

This analysis focuses on testing a commonly used structural parameter identification scheme, that claims to identify fundamental and non-fundamental components of stock prices. In particular, five related structural models, which are widely used in the literature on assessing stock price determinants are considered. Each of these models consist of three variables. The first variable represents different proxies of economic activity such as real GDP, the industrial production index, real dividends and real earnings; each proxy being a different model. All models are either specified in vector error correction (VEC) or in vector autoregressive (VAR) form. Restrictions are placed on the long-run effects matrix as in Blanchard and Quah (1989), making it lower triangular. All models are hence just-identified in the traditional sense.

A Markov switching in heteroskedasticity model as in Lanne et al. (2010) and Herwartz and Lütkepohl (2011) is used to test whether the long-run restrictions are supported by the data. It is found that for two of the models considered, the long-run identification scheme 
appropriately classifies shocks as being either fundamental or non-fundamental. Those are the models with real GDP and real dividends as proxies of real economic activity.

Three robustness tests are performed; one by removing very turbulent periods at the beginning and at the end of the sample. Another by allowing for a switching intercept term in addition to the switching covariance matrix; and a final robustness test uses the S\&P 500 index instead of the DJIA 30 index to represent the stock price. The robustness tests largely confirm the original findings.

Therefore, even though all the models are similar in the sense of using a different proxy for economic activity, results of this paper suggest that simply using the same identification scheme for models with different variables may not be warranted by the data. Structural shocks may not be properly identified in this way, making any labeling of the shocks ambiguous. Hence, in order to ensure that economic shocks of interest are captured, it is important to test the relevant identification scheme using the Markov switching in heteroskedasticity framework.

This paper therefore finds that models in which real GDP and real dividends are used as proxies for economic activity could potentially capture fundamental and non-fundamental shocks to stock prices. Since the findings in this paper are relatively robust, they serve as a good guideline when conducting future research in this field.

\section{References}

DE Allen and W. Yang. Do uk stock prices deviate from fundamentals? Mathematics and computers in simulation, 64(3):373-383, 2004.

G. Amisano and C. Giannini. Topics in structural VAR econometrics. Berlin/Heidelberg, 1997.

M. Binswanger. Stock market booms and real economic activity: Is this time different? International Review of Economics \& Finance, 9(4):387-415, 2000.

M. Binswanger. How important are fundamentals? Evidence from a structural VAR model for the stock markets in the US, Japan and Europe. Journal of International Financial Markets, Institutions \& Money, 14(2):185-201, 2004a.

M. Binswanger. How do stock prices respond to fundamental shocks? Finance Research Letters, 1(2):90-99, 2004b.

M. Binswanger. Stock returns and real activity in the g-7 countries: Did the relationship change during the 1980s? The Quarterly Review of Economics and Finance, 44(2):237-252, 2004c. 
O.J. Blanchard and D. Quah. The dynamic effects of aggregate demand and supply disturbances. The American Economic Review, 79(4):655-673, 1989.

H. Chung and B.S. Lee. Fundamental and nonfundamental components in stock prices of pacific-rim countries. Pacific-Basin Finance Journal, 6(3):321-346, 1998.

N. Groenewold. Fundamental share prices and aggregate real output. Applied Financial Economics, 14(9):651-661, 2004.

J.D. Hamilton. Time series analysis. Princeton Univ Pr, 1994.

H. Herwartz and H. Lütkepohl. Structural vector autoregressions with markov switching: Combining conventional with statistical identification of shocks. 2011.

R. Jean and T. Eldomiaty. How do stock prices respond to fundamental shocks in the case of the United States? Evidence from NASDAQ and DJIA. The Quarterly Review of Economics and Finance, 50(3):310-322, 2010. ISSN 1062-9769.

S. Johansen. Likelihood-based inference in cointegrated vector autoregressive models. Oxford University Press, USA, 1995. ISBN 0198774508.

R. King, C.I. Plosser, J.H. Stock, and M.W. Watson. Stochastic trends and economic fluctuations, 1992.

HM Krolzig. Markov-Switching Vector Autoregressions-Modelling. Statistical Inference and Application to Business Cycle Analysis, 1997.

M. Lanne and H. Lütkepohl. Structural vector autoregressions with nonnormal residuals. Journal of Business \& Economic Statistics, 28(1):159-168, 2010.

M. Lanne, H. Lütkepohl, and K. Maciejowska. Structural vector autoregressions with markov switching. Journal of Economic Dynamics and Control, 34(2):121-131, 2010.

N.T. Laopodis. Are fundamentals still relevant for european economies in the post-euro period? Economic Modelling, 26(5):835-850, 2009.

B.S. Lee. Fundamentals and bubbles in asset prices: Evidence from us and japanese asset prices. Asia-Pacific Financial Markets, 2(2):89-122, 1995a.

B.S. Lee. Permanent, temporary, and non-fundamental components of stock prices. Journal of Financial and Quantitative Analysis, 33:1-32, 1998. 
H. Lütkepohl. New introduction to multiple time series analysis. Springer, 2005. ISBN 3540401725 .

H. Lütkepohl and M. Krätzig, editors. Applied Time Series Econometrics. Cambridge University Press, Cambridge, 2004.

Z. Psaradakis and N. Spagnolo. Joint determination of the state dimension and autoregressive order for models with markov regime switching. Journal of Time Series Analysis, 27(5):753766, 2006.

D.E. Rapach. Macro shocks and real stock prices. Journal of Economics and Business, 53(1): 5-26, 2001.

P. Saikkonen and H. Lütkepohl. Testing for the cointegrating rank of a var process with structural shifts. Journal of business \& economic statistics, 18(4):451-464, 2000.

N.R. Swanson and C.W.J. Granger. Impulse response functions based on a causal approach to residual orthogonalization in vector autoregressions. Journal of the American Statistical Association, 92(437):357-367, 1997.

P.J.G. Vlaar. On the asymptotic distribution of impulse response functions with long-run restrictions. Econometric Theory, 20(05):891-903, 2004. ISSN 1469-4360. 